Research Paper

\title{
Vasculotide restores the blood-brain barrier after focused ultrasound-induced permeability in a mouse model of Alzheimer's disease
}

\author{
Madelaine Lynch ${ }^{1,2}$, Stefan Heinen ${ }^{1}$, Kelly Markham-Coultes ${ }^{1}$, Meaghan O’Reilly ${ }^{3,4}$, Paul Van Slyke ${ }^{5}$, Daniel J. \\ Dumont ${ }^{1,4 \#, ~ K u l l e r v o ~ H y n y n e n ~}{ }^{3,4}$, Isabelle Aubert ${ }^{1,2} \llbracket$ \\ 1. Biological Sciences, Sunnybrook Research Institute, 2075 Bayview Ave. Toronto, ON, Canada M4N 3M5. \\ 2. Laboratory Medicine \& Pathobiology, University of Toronto, 27 King's College Circle, Toronto, ON, Canada, M5S 1A1. \\ 3. Physical Sciences, Sunnybrook Research Institute, 2075 Bayview Ave. Toronto, ON, Canada M4N 3M5. \\ 4. Medical Biophysics, University of Toronto, 101 College Street, Toronto, ON, Canada, M5G 1L7. \\ 5. Vasomune Therapeutics, 661 University Ave \#465, Toronto, ON M5G 1M1. \\ \#In memory of Daniel J. Dumont, deceased. \\ $\bowtie$ Corresponding author: Sunnybrook Research Institute, 2075 Bayview Ave. S112, Toronto ON, Canada, M4N 3M5 Tel. +1 416 480 5831 isabelle.aubert@sri.utoronto.ca (I. \\ Aubert).
}

(C) The author(s). This is an open access article distributed under the terms of the Creative Commons Attribution License (https://creativecommons.org/licenses/by/4.0/). See http://ivyspring.com/terms for full terms and conditions.

Received: 2020.06.12; Accepted: 2020.10.28; Published: 2021.01.01

\begin{abstract}
Focused ultrasound (FUS) is used to locally and transiently induce blood-brain barrier (BBB) permeability, allowing targeted drug delivery to the brain. The purpose of the current study is to evaluate the potential of Vasculotide to accelerate the recovery of the BBB following FUS disruption in the TgCRND8 mouse model of amyloidosis, characteristic of Alzheimer's disease (AD). Accelerating the restoration of the BBB post-FUS would represent an additional safety procedure, which could be beneficial for clinical applications.

Methods: TgCRND8 mice and their non-transgenic littermates were treated with Vasculotide $(250 \mathrm{ng}$, intraperitoneal) every 48 hours for 3 months. BBB permeability was induced using FUS, in presence of intravenously injected microbubbles, in TgCRND8 and non-transgenic mice, and confirmed at time 0 by MRI enhancement using the contrast agent gadolinium. BBB closure was assessed at 6,12 and 20 hours by MRI. In a separate cohort of animals, BBB closure was assessed at 24-hours post-FUS using Evans blue injected intravenously and followed by histological evaluation.

Results: Chronic Vasculotide administration significantly reduces the ultra-harmonic threshold required for FUS-induced BBB permeability in the TgCRND8 mice. In addition, Vasculotide treatment led to a faster restoration of the BBB following FUS in TgCRND8 mice. BBB closure after FUS is not significantly different between TgCRND8 and non-transgenic mice. BBB permeability was assessed by gadolinium up to 20-hours post-FUS, demonstrating $87 \%$ closure in Vasculotide treated TgCRND8 mice, as opposed to $52 \%$ in PBS treated TgCRND8 mice, $58 \%$ in PBS treated non-transgenic mice, and $74 \%$ in Vasculotide treated non-transgenic mice. In both TgCRND8 mice and non-transgenic littermates the BBB was impermeable to Evans blue dye at 24-hours post-FUS.

Conclusion: Vasculotide reduces the pressure required for microbubble ultra-harmonic onset for FUS-induced BBB permeability and it accelerates BBB restoration in a mouse model of amyloidosis, suggesting its potential clinical utility to promote vascular health, plasticity and repair in AD.
\end{abstract}

Key words: Vasculotide, blood-brain barrier, transcranial focused ultrasound, Alzheimer's disease

\section{Introduction}

Transcranial focused ultrasound (FUS) with microbubbles is a minimally invasive technique used to transiently increase the permeability of the bloodbrain barrier (BBB) [1]. FUS can destabilize the BBB at the level of tight junction proteins, which gradually return to baseline levels 4 hours post-FUS [2,3]. To date, the safety profile of FUS has been established in healthy animals, including mice [4], rats [2], rabbits [1] and rhesus macaques $[5,6]$, while the primary clinical use of FUS would be in disease states, in which the 
BBB may have a compromised restorative capacity. For example, Alzheimer's disease (AD) has significant cerebrovascular pathology, including cerebral amyloid angiopathy (CAA) $[7,8]$, raising questions around whether the plasticity of the $\mathrm{BBB}$ and its capacity for repair in response to FUS is compromised $[8,9]$.

FUS alone, and in combination with therapeutics has highlighted its potential in the treatment of $\mathrm{AD}$ [10-14]. A recent safety clinical trial conducted in a small group of $\mathrm{AD}$ patients suggests that functioning of the BBB is restored within 24 hours following FUS [15]. To further improve safety and possibly treatment efficacy, promoting a healthy vasculature prior to the administration of FUS may have significant benefits. This could avoid the potential prolonged exposure of the brain to blood components, which could be detrimental. The ability to promote a rapid restoration of the BBB after FUS may significantly contribute to better treatment options for AD patients in the future. As such, we explored the potential of Vasculotide to promote BBB restoration after FUS.

Vasculotide is a $14 \mathrm{kDa}$ angiopoietin-1 (Ang-1) peptide mimetic composed of 4 short synthetic peptides (CHHHRHSF) engineered on a tetrameric polyethylene glycol (PEG) backbone [16,17]. The impact of Vasculotide on the brain vasculature has been understudied. Recent data in a model of diabetic stroke demonstrates that Vasculotide has neuroprotective properties including protection from BBB breakdown and significant reductions in markers of neuroinflammation [18]. These improvements correlated with increased functioning when assessed by blinded observer for neurological severity score (NSS) [18]. In organs other than the brain, Vasculotide was found to decrease vascular endothelial cell permeability in sepsis, endotoxemia, ionizing radiation damage, tumor cell extravasation, influenza, acute kidney injury, dermatitis, pneumonia and hemorrhagic shock $[16,17,19-26]$.

Here, we first compared the closure of the BBB following FUS-induced permeability in a transgenic mouse model of AD (TgCRDN8) with characteristic amyloid pathology [27] to the $\mathrm{BBB}$ response in healthy, non-transgenic littermates. We then assessed the ability of Vasculotide to improve the restoration of the BBB following FUS-induced permeability in TgCRND8 and non-transgenic littermates.

Our findings support the potential of Vasculotide to reduce the time required to reseal the BBB following FUS treatments; a therapeutic approach which is being evaluated in several neurodegenerative disorders including $\mathrm{AD}$ and amyotrophic lateral sclerosis (ALS) $[15,28,29]$. Our study can have broader implication for Vasculotide as a therapeutic of interest in disease states presenting with a compromised $\mathrm{BBB}$, including stroke and vascular dementia.

\section{Materials and Methods}

\section{Vasculotide}

Drs. Daniel Dumont and Paul Van Slyke at Sunnybrook Research Institute generously provided Vasculotide. Vasculotide was prepared as described by David et al. [22]. Briefly, a modified T7 peptide (CHHHRHSF) was covalently linked to a four-armed polyethylene glycol (PEG)-maleimide backbone to generate the compound, Vasculotide. Vasculotide was reconstituted in phosphate buffered saline at a concentration of $1 \mathrm{mg} / \mathrm{ml}$ and diluted to a working concentration of $2.5 \mathrm{ng} / \mathrm{ul}$.

\section{Animal Care and Husbandry}

Transgenic mice from the Centre for Research in Neurodegenerative Disease (TgCRND8) and their non-transgenic littermates [27] were bred and housed at Sunnybrook Research Institute. TgCRND8 mice are on a mixed $\mathrm{C} 57 / \mathrm{C} 3 \mathrm{H}$ background and have a double mutation in the amyloid precursor protein 695 (KM670/671NL, V717F) [27]. These mice present with amyloid pathology and cognitive deficits by 3 months of age [27]. TgCRND8 mice develop CAA, detectable at 4 months of age and increasingly evident by 5-6 months of age [7].

\section{Treatment}

Three-month-old male and female mice were injected with Vasculotide (250 ng, intraperitoneal), or PBS control every 48 hours starting at 3 months of age ( $\mathrm{n}=6$ per group; non-Tg PBS, non-Tg VT, TgCRND8 PBS, TgCRND8 VT). Treatment paradigm was chosen based on CAA pathology [7]. Treatment started at 3 months (pre-CAA) and ended at 5-6 months, when CAA is established [7], after which mice underwent MRIgFUS (Table 1). For MRIgFUS, mice were anesthetized with isofluorane, depilated and fitted with a tail vein catheter. Mice were placed supine on an MRIgFUS compatible positioning system fitted for the ultrasound transducer and the 7T MRI (BioSpec 70/30 USR, Bruker, Billerica, Mass). Mice were deeply anesthetized using a cocktail of $15 \%$ ketamine and 5\% xylazine, perfused transcardially with $0.9 \%$ saline and sacrificed 24 hours after MRIgFUS. All animal procedures were conducted with the approval of the Animal Care Committee of Sunnybrook Research Institute and in compliance with the guidelines established by the Canadian Council on Animal Care and the Animals for Research Act of Ontario. 
Table 1. Experimental Design

\begin{tabular}{|c|c|c|c|c|}
\hline & \multicolumn{3}{|l|}{ Mice } & \multirow{2}{*}{ Analysis } \\
\hline & Start Age & Treatment Groups (n) & End Age & \\
\hline \multirow[t]{4}{*}{ Chronic vasculotide (VT) } & 3 months & non-Tg-VT (6) & 5-6 months & Post-FUS: At 6, 12, 20-hrs \\
\hline & & non-Tg-PBS (6) & & BBB permeability monitored by MRI (gadolinium); \\
\hline & & TgCRND8-VT (6) & & Sacrificed at $24-\mathrm{hrs}$. \\
\hline & & TgCRND8-PBS (6) & & \\
\hline \multirow[t]{3}{*}{ BBB closure to Evans blue (EB) } & 7 months & EB 0-hrs post-FUS: non- $\operatorname{Tg}(1)$ & 7 months & Brain cut at $1 \mu \mathrm{m}$; optical EB imaging. \\
\hline & & EB 6-hrs post-FUS: non-Tg (1) & & Brain cut at $500 \mu \mathrm{m}$; optical EB imaging. \\
\hline & 5 months & $\begin{array}{l}\text { EB 24-hrs post-FUS: } \\
\text { non- } \operatorname{Tg}(6) \text {; TgCRND8 (4) }\end{array}$ & 5 months & Brains cut at $500 \mu \mathrm{m}$; optical EB imaging. \\
\hline
\end{tabular}

\section{MR Imaging}

T2-weighted MR imaging was used for targeting of FUS to four spots on the left hemisphere. Contrast-enhanced $(0.1 \mathrm{ml} / \mathrm{kg}$, Omniscan, GE) T1-weighted images $(\mathrm{TR} / \mathrm{TE}=500 / 10 \mathrm{msec}$ ) were used to assess BBB permeability immediately after FUS, as described in [30], as well as at 6, 12 and 20 hours post-FUS. Contrast was given as a bolus intravenous injection immediately after FUS. At 6, 12 and 20 hours post-FUS contrast was given as an intraperitoneal injection $(0.5 \mathrm{ml} / \mathrm{kg}) 20$ minutes prior to image acquisition, and MR imaging was performed for 15 minutes to ensure enhancement peak was detected.

\section{Focused Ultrasound}

Focused ultrasound was generated using an in-house fabricated lead zirconate titanate (PZT) transducer (PZT from DeL Piezo Specialties, LLC, West Pal Beach, Florida, USA), with a $75 \mathrm{~mm}$ diameter and $60 \mathrm{~mm}$ focal length (focal-number $=0.8$ ). Sonications consisted of $10 \mathrm{~ms}$ bursts at a frequency of $1.68 \mathrm{MHz}$ and $1 \mathrm{~Hz}$ pulse repetition frequency (PRF) for a total of 2 minutes. Ultrasound was targeted to four focal spots on the left hemisphere, with the contralateral hemisphere serving as an untreated control (Fig. 1A). At the time of sonication, mice received an intravenous injection of $0.02 \mathrm{ml} / \mathrm{kg}$ Definity microbubbles, diluted $1: 50$ in saline (Lantheus Medical Imaging, Billerica, Mass, USA) to induce BBB permeability. A custom-built PVDF hydrophone was used to detect microbubble emissions during ultrasound bursts [31]. The burst pressure was modulated based on the appearance of sub-harmonic $\left(0.5 f_{0}\right)$ and ultra-harmonic $\left(1.5 f_{0}\right)$ signals via a real-time control algorithm (Fig. 1B) [32]. Pressure was derated to account for the insertion loss of the skull bone $(18 \%)$ and attenuation through $2.5 \mathrm{~mm}$ of brain tissue at $5 \mathrm{~Np} / \mathrm{m} / \mathrm{MHz}[1,33]$. The pressure was increased from $0.2 \mathrm{MPa}$ in steps of 0.02 Mpa until ultra-harmonic signals were detected, at which point the pressure was decreased to $25 \%$ of the peak pressure reached for the remainder of the sonication. Pressure increases were recorded for each independent focal spot every second (Fig. 2). Each of the four focal spots were monitored independently, thus generating four unique time-pressure signatures. This demonstrates the step-wise pressure increase every second until the detection of local ultraharmonic frequencies (peak pressure), after which the feedback mechanism triggers the pressure at each focal spot to be reduced to $25 \%$ of the peak pressure for the remainder of the sonication.

\section{Quantification of blood-brain barrier permeability}

\section{MATLAB Analysis of Blood-Brain Barrier Closure}

To assess BBB closure mice received intraperitoneal injections of gadolinium contrast agent, Omniscan $(0.5 \mathrm{ml} / \mathrm{kg})$, which circulated 20 minutes prior to image acquisition. For the purpose of this study longitudinal intravenous injections in the mouse tail vein were a technical limitation. A catheter cannot be left in for extended period of time, nor could the tail vein be repeatedly injected within the short time frame. Upon inducing BBB permeability, the initial enhancement and coordinates of each focal spot were recorded. In subsequent follow-up images the same coordinates were used to quantify enhancement (Fig. 1C); BBB closure was defined when there was less than two standard deviations of the enhanced relative to the unenhanced hemisphere. Each focal spot represents a distinct vasculature composition and a unique acoustic emission, as detected by the feedback controller. As such, each focal spot was considered independently. Gadolinium contrast enhancement was quantified in MATLAB (Mathworks, Natick, MA) by finding the mean pixel intensity of a $3 \times 3$ voxel region of interest surrounding the point of maximum enhancement.

\section{Evans Blue Quantification}

24-hours after MRIgFUS, a separate cohort of TgCRND8 $(n=4)$ and non-Tg $(n=6)$ mice received an intravenous injection of $4 \mathrm{ml} / \mathrm{kg}$ of $2 \%$ Evans blue (EB) dye (Table 1). The dye was allowed to circulate 
10 minutes prior to sacrifice. Animals were deeply anesthetized using a cocktail of $15 \%$ ketamine and 5\% xylazine and perfused transcardially with $0.9 \%$ saline followed by $4 \%$ paraformaldehyde. Brains were isolated, post-fixed in $4 \%$ paraformaldehyde for 24 hours then transferred to $30 \%$ sucrose.

The ten 24-hour and one 6-hour, post-FUS brains were cut on a vibratome at $0.5 \mathrm{~mm}$ for optical imaging using a Xenogen IVIS 200. One brain was collected immediately after FUS and cut at $1 \mathrm{~mm}$ for optical imaging. Fluorescence data was collected using the Cy5.5 filter with an exposure time of $0.5 \mathrm{~s}$. Known quantities of EB were spotted onto filter paper to create a standard curve, allowing quantification of EB from regions of interest within the brain as described by Jaffer et al. [34].

\section{Immunohistochemistry}

Brains for immunohistochemistry were cut axially at a thickness of $40 \mu \mathrm{m}$. Free floating sections were washed 3 times in PBS $+0.3 \%$ Triton X100, pH 7.4 prior to incubation in blocking solution (PBS, $3 \%$ BSA, $0.3 \%$ Triton X100, 3\% donkey serum) for 3 hours at room temperature.

Primary and secondary antibodies were incubated in blocking solution overnight at $4^{\circ} \mathrm{C}$. Fibrinogen was stained using the polyclonal anti-human fibrinogen antibody (Dako, A0080, 1:100). Erythrocytes were stained using the rat anti-mouse Ter119 antibody (BD Pharmingen, 553671, 1:100). Mouse IgG was stained and detected using the donkey anti-mouse IgG-Cy5 antibody (Jackson ImmunoResearch, 715-175-150, 1:200). Staining for
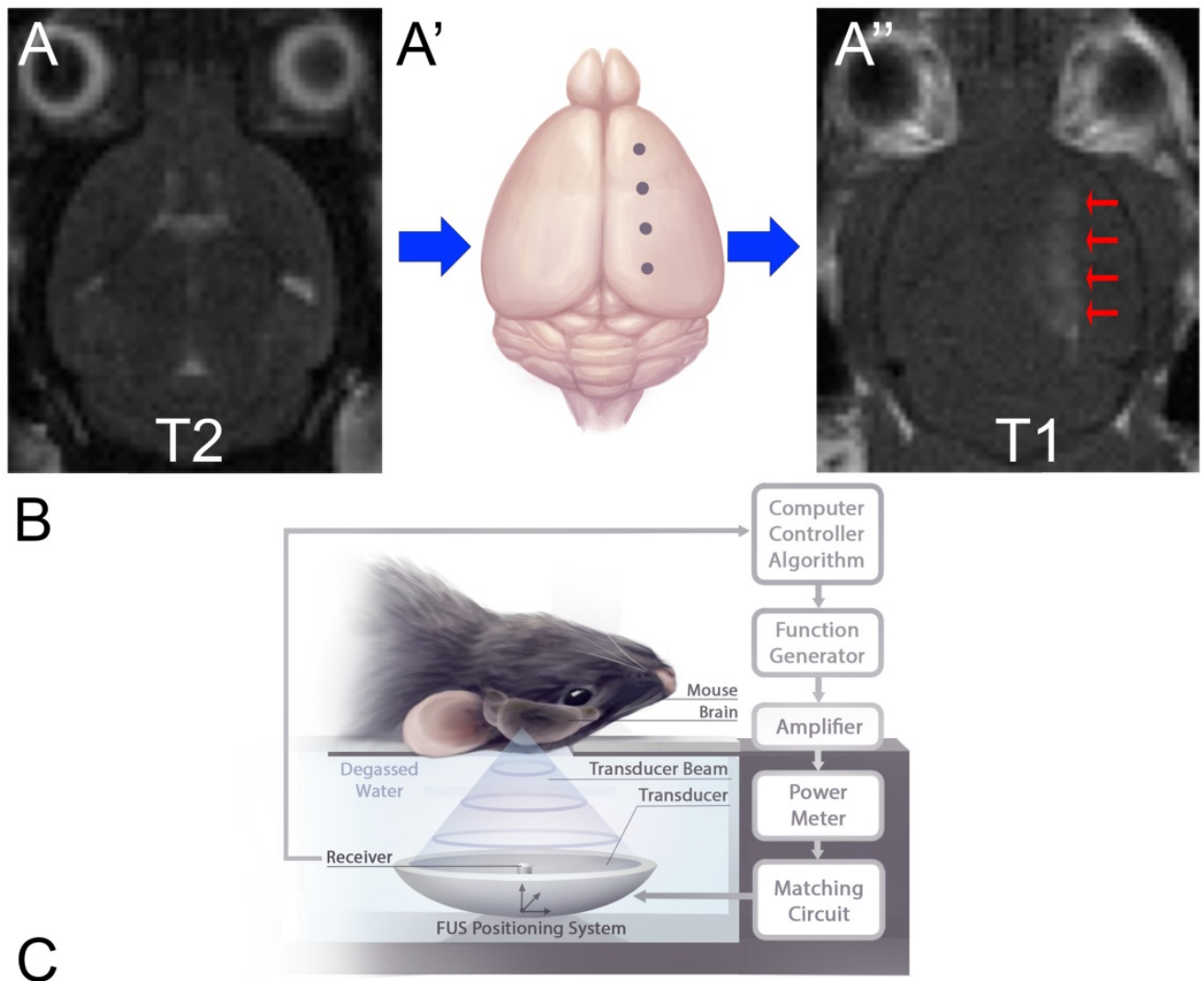

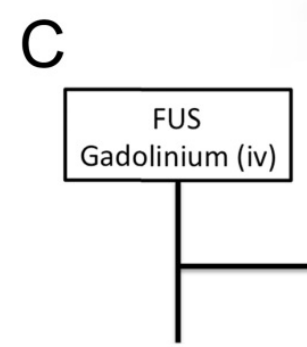

Time (hrs) 0

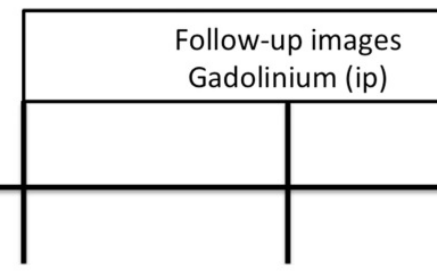

12

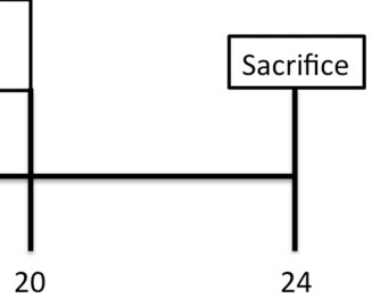

Figure 1. Experimental Setup and Schematic of Treatment. (A) Magnetic Resonance Imaging (MRI) targeting. Mice were placed in a supine position on an MRI-compatible sled. (A) T2-weighted images were acquired for targeting of FUS beam to four spots along one hemisphere ( $A^{\prime}$ ). ( $A^{\prime \prime}$, arrows) Blood-brain barrier (BBB) permeability was confirmed by gadolinium enhancement using T1-weighted images. (B) Schematic of FUS treatment. Mice remained on the MRI-compatible sled and were placed above the transducer for focused ultrasound (FUS) treatment. Briefly, FUS is generated from a transducer, immersed in a water bath, positioned under the mouse. Microbubble emissions are detected by a receiver, which feeds back to a computer controller algorithm to modulate FUS power. (C) Experimental timeline. FUS treatment occurred at 0 -hour and BBB permeability was confirmed by MRI using an intravenous injection of gadolinium contrast agent. Subsequently images were acquired 6 , 12 and 20 hours after FUS using intraperitoneal injections of gadolinium contrast agent. Mice were then sacrificed at 24-hours. 
fibrinogen and erythrocytes was detected using donkey anti-rabbit-Cy3 antibody (Jackson ImmunoResearch, 711-162-152, 1:500) and donkey anti-rat 488 (Jackson ImmunoResearch, 712-545-150, 1:500), respectively. Whole sections were imaged on the TissueScope 4000 fluorescence tissue scanner (Huron Digital Pathology) at a scan resolution of 0.5 $\mu \mathrm{m} /$ pixel and constant PMT settings.

\section{Statistical Analysis}

All statistical analysis was done using GraphPad Prism. Enhancement and BBB disruption pressure were analyzed using a 2-way ANOVA with a Tukey post-hoc test. Restoration of the BBB was analyzed by log-rank (Mantel-Cox). Multiple comparisons amongst BBB restoration curves were done individually (4 comparisons), and corrected for multiple comparisons using the Bonferroni corrected threshold; the significance level of 0.05 was divided by the number of comparisons (4) resulting in a multiple comparisons significance threshold of 0.0125 . Multiple comparisons in which $p<0.0125$ were considered statistically different. Significance is

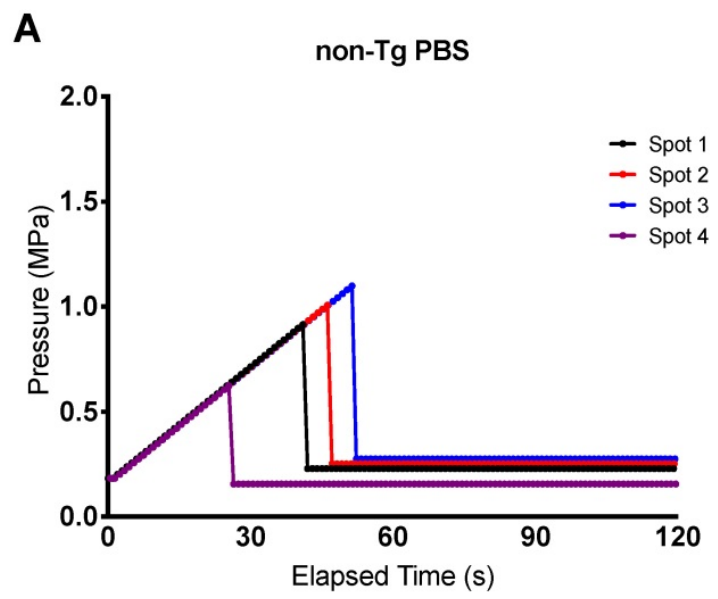

\section{C}

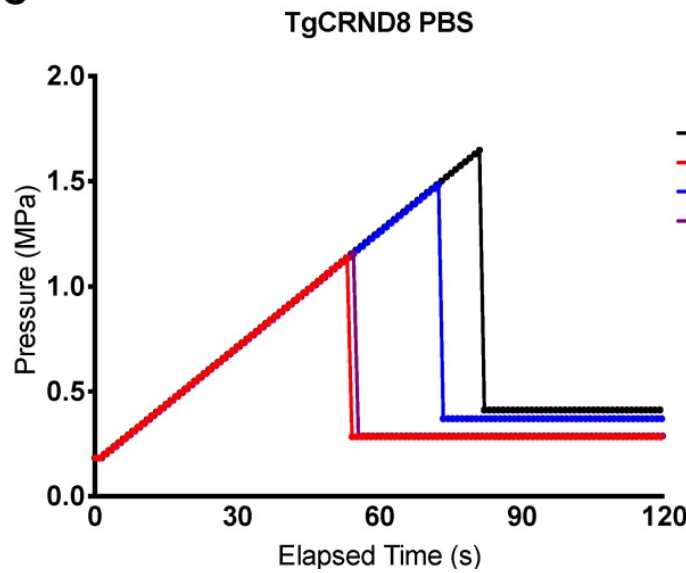

defined as: ${ }^{*} p<0.05,{ }^{* *} p<0.01,{ }^{* * *} p<0.001$.

\section{Results}

\section{The blood-brain barrier is impermeable to Evans blue 24-hours post-FUS}

In our experimental design gadolinium was injected intraperitoneally to detect contrast in the brain following FUS. This led to prolonged detection of BBB permeability, as evidenced by gadolinium detection at the 20-hour time point (Fig. 3). While this is within the range of closure reported in other studies $[35,36]$, most investigators have used intravenous gadolinium [30], Evans blue [2] and other imaging metrics such as electron microscopy [37] to define BBB closure. Here, we sought to corroborate these previous closure findings, independently of Vasculotide. This was done in a separate cohort of mice by EB intravenous injection $(2 \%, 4 \mathrm{ml} / \mathrm{kg})$ at 6 and 24-hrs post-FUS, brain isolation and quantitative EB fluorescent detection (Fig. 3D, F).

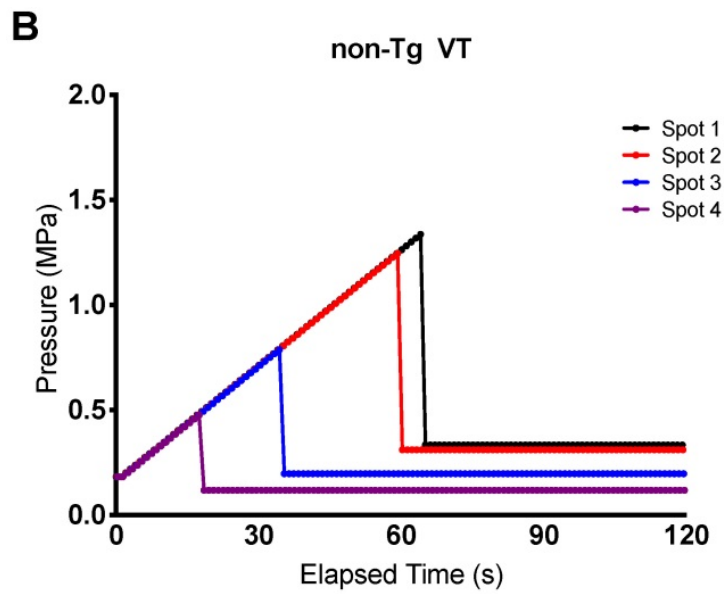

D

TgCRND8 VT

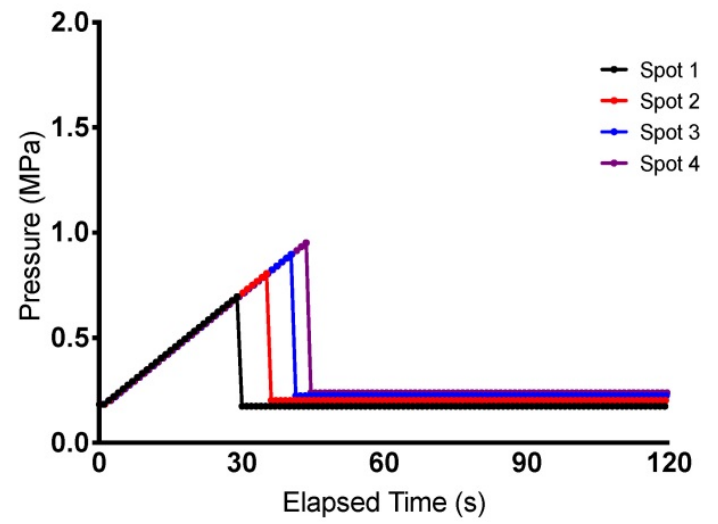

Figure 2. Pressure drop to $\mathbf{2 5 \%}$ of peak pressure to induce blood-brain barrier permeability. Focused ultrasound pressure increased in steps of $0.02 \mathrm{MPa}$ every second until reaching peak pressure at which point ultra-harmonic signals were detected. The pressure is subsequently reduced to $25 \%$ of the peak pressure and maintained to induce blood-brain barrier permeability. Each spot of the four focal spots were monitored independently thereby generating a unique time and peak pressure signature. Graphs are a representative mouse of each genotype and treatment, i.e. (A) non-Tg PBS (B) non-Tg VT (C) TgCRND8 PBS (D) TgCRND8 VT, demonstrating pressure increase (MPa) on the $y$-axis and time (seconds) on the x-axis. 


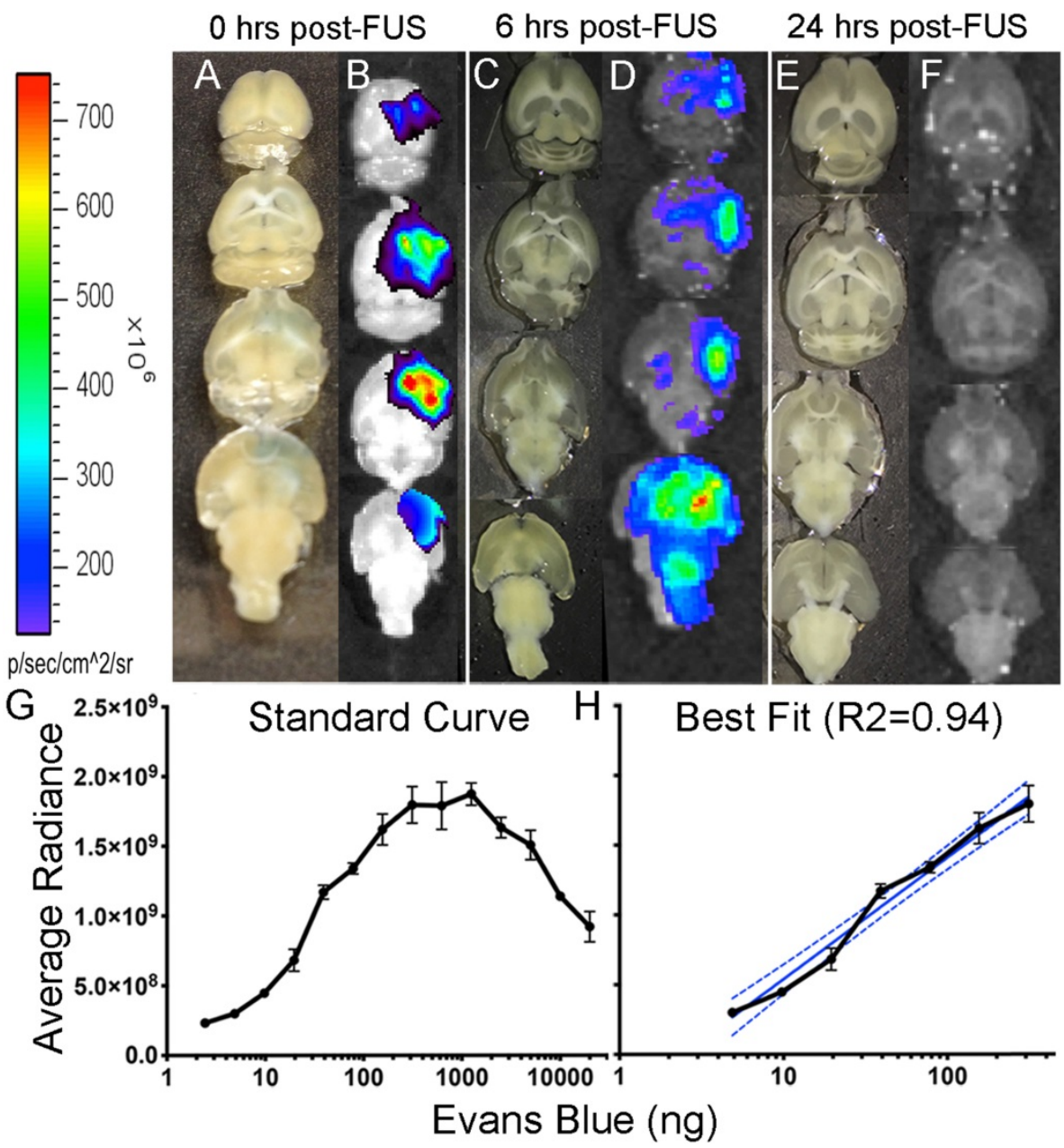

Figure 3. Detection of Evans blue after focused ultrasound-induced blood-brain barrier permeability. Images of Evans blue dye in brain cut at 1 mm, 0 -hours after FUS (A), in which $162 \mathrm{ng}$ of Evans blue were detected by optical imaging (B); Images of brain cut at 500 m, 6-hours after FUS (C), in which 33 ng of Evans blue were detected by optical imaging; Images of brains cut at $500 \mu \mathrm{m}, 24$-hours after FUS (E) in which no Evans blue was detected by optical imaging in both non-Tg (shown here) and TgCRND8 mice (F). Standard curve was created by fluorescent detection of known quantities (ng) of Evans blue using a Xenogen IVIS 200 Optical Imaging System. The quantity of Evans blue was plotted logarithmically relative to the average radiance (a measure of photons/pixel) $(G)$. A semi-log line of best fit $\left(R^{2}=0.94\right)$ was fitted to the linear portion of curve " $C$ " and used to interpolate Evans blue quantities $(\mathrm{H})$. Colour scale was generated in relation to the images in panel " $D$ ".

Known quantities of EB were used to generate the logarithmic standard curve (Fig. 3G). From this curve, a semi-log line was fitted to the linear portion $\left(\mathrm{R}^{2}=0.94 ;\right.$ Fig. $\left.3 \mathrm{H}\right)$ to interpolate the quantities (ng) of EB. Quantification of EB immediately after FUS revealed $162 \mathrm{ng}$ of EB (Fig. 3B) in the brain and only 33 ng of EB 6-hours after FUS (Fig. 3D). EB injections 24-hours after FUS did not show any EB visible by eye or detected by fluorescence in TgCRND8 or non-Tg mice (Fig. 3F). The lack of EB post-FUS indicates that the $\mathrm{BBB}$ is impermeable at 24-hours in both non-Tg and TgCRND8 mice.

\section{Vasculotide reduces the ultra-harmonic threshold required for blood-brain barrier disruption in TgCRND8 mice}

Our method for the application of FUS uses a control algorithm to safely modulate the pressure required to induce BBB permeability. Microbubble acoustic emissions are detected after each burst, which facilitates the stepwise increase in applied pressure. Detection of sub-harmonic or ultraharmonic emission is regarded as the upper safety limit, after which the program reduces the applied pressure to the predetermined level of $25 \%$ to induce BBB permeability [32].

Analysis of the maximum pressure reached by the ultrasound transducer demonstrated no statistical difference in the maximum threshold required to induce sub- and ultra-harmonic bubble behaviour between TgCRND8 and non-Tg mice. Vasculotide treatment had no effect on the threshold required in non-Tg mice. However, TgCRND8 mice treated with Vasculotide (TgCRND8-VT) had a 21-29\% lower threshold to induce sub- and ultra-harmonic bubble behaviour when compared to all other groups (Fig. 4, TgCRND8-VT compared to TgCRND8-PBS $p=0.03$; non-Tg-VT, $p=0.01$; non-Tg-PBS, $p=0.0002)$. A Grubbs test was conducted and demonstrated no significant outliers. 


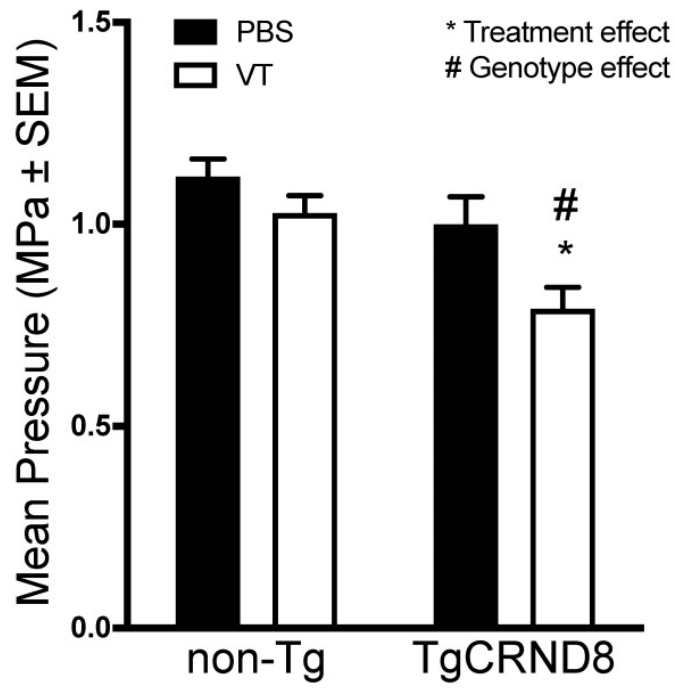

Figure 4. Vasculotide reduces the threshold for sub- and ultra-harmonic bubble behaviour in TgCRND8 mice. Focused ultrasound transducer detects sub-harmonic frequencies of injected microbubbles, gradually increasing pressure to induce blood-brain barrier (BBB) permeability until sub-harmonic emissions are detected at which point the pressure is reduced to $25 \%$ and maintained to induce BBB permeability. Maximum pressures were recorded for each focal spot. Data presented as mean+SEM, N=24 focal spots per group. Statistical analysis was done using a 2-way ANOVA with a Tukey's multiple comparisons post-test. \# genotype effect * treatment effect as calculated by Tukey's multiple comparisons.

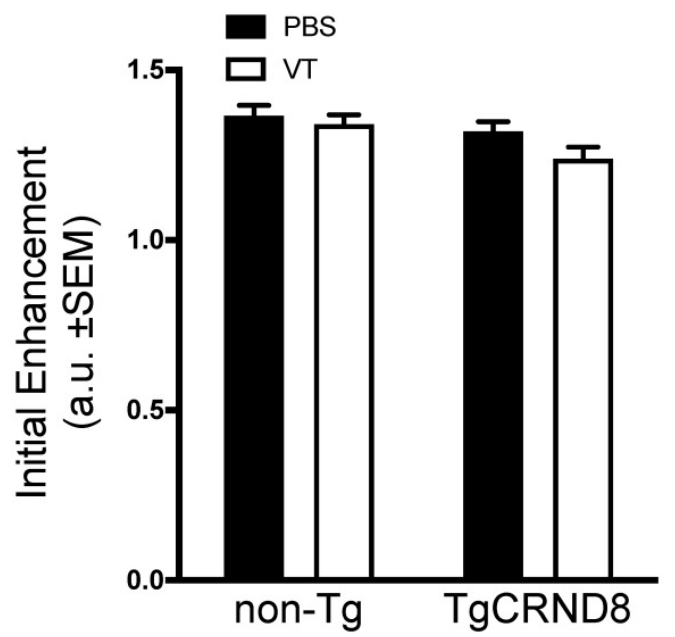

Figure 5. Vasculotide does not impact the initial enhancement after focused ultrasound in TgCRND8 mice. Gadolinium enhancement immediately after focused ultrasound -mediated blood-brain barrier permeability was quantified by the intensity of a $3 \times 3$ voxel region using a custom program in MATLAB. Data presented as the ratio of gadolinium enhancement between the enhanced and unenhanced hemispheres as mean + SEM, N=21-24 focal spots per group. Statistical analysis was done using a 2-way ANOVA with a Tukey multiple comparisons post-test.

\section{Vasculotide does not impact the initial enhancement in TgCRND8 mice}

Immediately after FUS sonication, mice received an intravenous dose of gadolinium to confirm BBB permeability. Quantification of gadolinium contrast revealed no significant difference in initial enhancement between TgCRND8 and non-Tg mice. Analysis of Vasculotide treated mice did not demonstrate any significant enhancement differences in either non-Tg or TgCRND8 mice (Fig. 5).

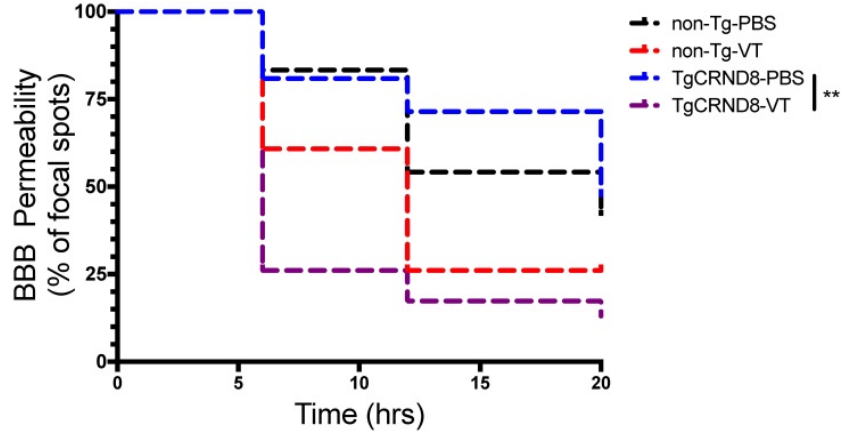

Figure 6. Vasculotide promotes blood-brain barrier restoration in TgCRND8 mice after focused ultrasound-induced permeability. Results are expressed as the percentage of focal spots permeable to gadolinium at 6,12 and 20 hours after focused ultrasound (i.e. at time $0,100 \%$ of focal spots are permeable). Closure was defined as less than two standard deviations between the enhanced and unenhanced hemisphere. Statistical analysis was done using a log rank (Mantel-Cox) test. Multiple comparisons were calculated at a Bonferroni corrected threshold of 0.0125 to determine significance. $N=21-24$ focal spots per line, $* * p<0.01$, $* * * p<0.001$.

\section{Vasculotide accelerates the closure of the blood-brain barrier post-FUS}

In TgCRND8 mice, Vasculotide treatment significantly reduced BBB closure time post-FUS, as indicated by the fewer number of focal spots permeable to gadolinium within the 20-hour time frame (Fig. 6, solid black line TgCRND8-VT). Specifically, the restoration of the BBB in Vasculotide treated TgCRND8 mice is indicated by the drop in the number of foci where gadolinium was detected at time zero $(100 \%)$ of FUS-induced BBB opening to $26 \%$, $17 \%$ and $13 \%$ of foci remained permeable to gadolinium at 6, 12 and 20 hours, respectively (Fig. 6, black solid line). In Vasculotide treated non-Tg mice, approximately $61 \%, 26 \%$ and $26 \%$ of foci remained permeable to gadolinium at 6, 12 and 20 hours, respectively (Fig. 6, black dashed line). Log-rank analysis revealed a statistically significant difference between TgCRND8-VT and TgCRND8-PBS ( $p=0.001)$.

Comparison of non-Tg and TgCRND8 mice revealed no significant difference in gadolinium permeability with respect to genotype (Fig. 6). In non- $\mathrm{Tg}$ mice, $83 \%, 54 \%$ and $42 \%$ of foci remained permeable to gadolinium at 6, 12 and 20 hours, respectively (Fig. 6, red dashed line). In TgCRND8 mice, $81 \%, 71 \%$ and $48 \%$ of foci remained permeable to gadolinium at 6, 12 and 20 hours, respectively (Fig. 6 , red solid line).

Taken together these results indicate a substantial effect of Vasculotide in accelerating the closure of the BBB after FUS. The total number of spots closed by 20 hours post-FUS was substantially greater in Vasculotide treated TgCRND8 mice with $86 \%$ of foci closed compared to PBS treated TgCRND8 mice in which $52 \%$ of foci were closed (Fig. 6, black compared to red solid lines). 


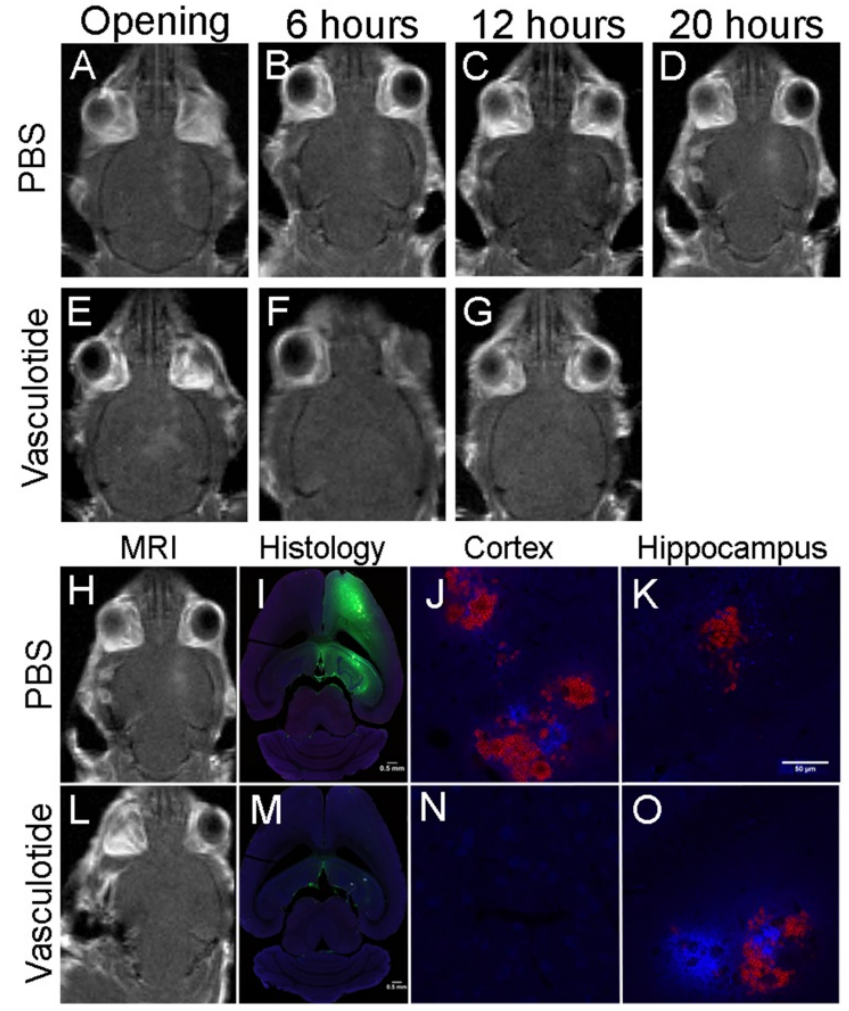

Figure 7. Gadolinium enhancement is consistent with blood-borne components extravasation at 24-hours. Representative images of PBS and Vasculotide treated TgCRND8 mice, which were defined (through gadolinium enhancement) as complete closure with Vasculotide and incomplete closure with PBS. Gadolinium enhancement in PBS treated TgCRND8 mouse at (A) opening, (B) 6-hours, (C) 12-hours and (D) 20-hours. Gadolinium enhancement in Vasculotide treated TgCRND8 mouse at (E) opening, (F) 6-hours and (G) 12-hours. (I, M) Histology sections 24-hours after FUS demonstrate $\lg$ (green) in locations consistent with gadolinium enhancement $(\mathrm{H}, \mathrm{L})$. Extravasation of fibrinogen (blue) and erythrocytes (red) are demonstrated in the 20X magnification images in the cortex (1, $\mathrm{N})$ and hippocampus $(\mathrm{K}, \mathrm{O})$. MR images were reflected right/left to match histology images.

\section{Extravasation of blood-borne components is consistent with prolonged gadolinium enhancement}

Gadolinium enhancement illustrated focal spots where BBB permeability was induced up to 20-hours post-FUS. Histological analysis of IgG (green), fibrinogen (blue) and erythrocyte (red) extravasation 24-hours post-FUS coincided with the locations of gadolinium permeability (Fig. 7). The extent of extravasation appears consistent with the rate of BBB closure. Qualitatively, mice that demonstrated rapid BBB restoration, such as the Vasculotide treated TgCRND8 mice (Fig. 7), had reduced entry of blood-borne molecules as compared to the PBS treated TgCRND8 mice (Fig. 7), which remained permeable to gadolinium.

\section{Discussion}

The ability of FUS to permeabilize the BBB for the delivery of therapeutics to the brain offers exciting new possibilities for the treatment of $\mathrm{AD}$ and other neurological diseases. Yet, important questions and potential concerns remain to be considered, such as how long the BBB remains permeable in presence of amyloid pathology and to what blood-borne molecules the brain will be exposed? As such, we investigated the timeline of the BBB closure after FUS in a mouse model with amyloid pathology, and whether BBB restoration could be accelerated with Vasculotide. Following FUS, BBB opening parameters, namely the pressure required for ultra-harmonic microbubble emissions, was reduced in Vasculotide-treated TgCRND8 mice. Additionally, Vasculotide treatment significantly accelerated BBB closure in TgCRND8 mice. Neither FUS-related BBB opening parameters nor closure differed between TgCRND8 and non-transgenic mice. While the presence of amyloid pathology alone does not alter FUS-induced BBB permeability, the results shown here indicate that the combination of amyloid pathology and Vasculotide treatment can influence FUS-induced BBB permeability and closure.

The presence of CAA in the TgCRND8 mice has been demonstrated as early as 4 months of age, causing changes in vascular morphology and functional deficits $[7,38]$. Previous research using FUS to open the $\mathrm{BBB}$, without the use of the feedback controller [32], required twice the dosage of microbubbles in TgCRND8 mice $(80 \mathrm{ul} / \mathrm{kg})$ as compared to non-transgenic littermates $(40 \mathrm{ul} / \mathrm{kg})$ in order to induce BBB permeability [11]. The requirement of twice the microbubble dosage at a constant pressure for FUS-induced BBB permeability, suggests an altered vascular response to FUS in TgCRND8 mice. Furthermore, after FUS, Burgess et al. showed a reduced permeability constant in TgCRND8 mice and an increase of slow leaking vessels attributed to the presence of CAA [39]. Despite differences in leakage kinetics, the acoustic pressure and probability of inducing BBB permeability remained the same between genotypes [39]. Therefore, in TgCRND8 mice although leakage occurs slower in response to FUS, the capacity for inducing BBB permeability is comparable to that of non- $\mathrm{Tg}$ mice [39]. Consistent with the study by Burgess et al. the results presented here show no disparities in acoustic pressure and permeability to gadolinium up to 20-hours after FUS between genotypes. This suggests that the TgCRND8 mice possess a similar capacity for repair as the non-Tg mice, facilitating safe application of FUS. Interestingly, Vasculotide reduced the upper ultra-harmonic threshold required to disrupt the BBB in the TgCRND8 mice. This suggests that the effect of Vasculotide in the TgCRND8 mice may alter the response of the vasculature to ultrasound exposure. Vasculotide treatment resulted 
in detection of sub- and ultra-harmonic emissions at lower pressures, suggesting that less pressure may be required to induce BBB permeability. Although the exact mechanism of Vasculotide in the brain has yet to be determined, it is possible that Vasculotide treatment improves vascular plasticity thereby making the cerebrovasculature more amenable to FUS-induced BBB permeability. Having shown a reduction in the ultra-harmonic threshold with no change in initial enhancement indicates that Vasculotide treatment can reduce the acoustic threshold required for BBB permeability without impeding the opening volume for potential therapeutic delivery.

Furthermore, Vasculotide treatment has a marked effect on reducing BBB permeability after FUS. In TgCRND8 mice chronic Vasculotide treatment not only increased the total number of focal spots impermeable to gadolinium within the 20-hour time frame, but also produced a more rapid restoration of the BBB. Previous studies have demonstrated the ability of acute Vasculotide treatment to improve endothelial barrier function and reduce vascular leakage in the lung and kidney $[17,40]$. Vasculotide has been shown to increase VE-cadherin, PECAM-1 and Sma1, likely contributing to the endothelial barrier improvement $[16,22]$. Therefore, it is plausible that Vasculotide produces a similar effect here in reducing brain endothelial cell permeability, possibly by increasing junction proteins or altering their cellular localization.

The results presented here suggest prolonged permeability to intraperitoneal gadolinium as long as 20-hours post-FUS. This permeability appears to be independent of genotype, as both non-Tg and TgCRND8 mice demonstrated extended gadolinium permeability. Research has shown that measurements evaluating the time it takes to restore the BBB after FUS is largely dependent on the molecular size of the tracer agent and FUS sonication parameters. Indeed, studies using different molecular size markers of BBB permeability, such EB ( $70 \mathrm{kDa})$, have cited BBB closure occurring 4-6 hours after FUS, with confirmation of closure at 24 hours, 72 hours and 4 weeks later $[1,2,30,37,41]$. Using MR contrast agents of various sizes (1-65 nm) Marty et al. demonstrated that the BBB is impermeable as rapidly as 90 minutes to the $7 \mathrm{~nm}$ P792, while Dotarem $(1 \mathrm{~nm})$ crossed the BBB up to 24 hours [36]. Using HRP (40 kDa) and lanthanum chloride (139 Da), Sheikov et al. showed closure of the BBB at both 6 and 24 hours [2]. However, using various exposure times of 6,8 and 10 $\mathrm{s}$ in rabbits, Mei et al. demonstrated the BBB remained permeable to gadolinium contrast agent as late as 24-hours after FUS [41]. Furthermore, Samiotaki et al. used various acoustic pressures $(0.30-0.60 \mathrm{MPa})$ and pulse lengths $(0.67 \mu \mathrm{s}-6.7 \mathrm{~ms})$ to demonstrate BBB permeability to gadolinium up to 72 hours post-FUS [35]. Similar to our study, Samiotaki used intraperitoneal injections of gadolinium at higher doses than the intravenous injections, which may allow greater sensitivity to small changes in the BBB [35,42]. The finding of $\mathrm{BBB}$ permeability at the 20-hour time point is relative to the delivery and size of gadolinium used. Studies from our labs and others [35,42] have supported the use of intraperitoneal gadolinium. However, the kinetics of this injection route differs from intravenous, which may thus be the source of prolonged enhancement. Work by Choi et al. [4] used intraperitoneal gadolinium, suggesting a slower time course of entry than intravenous and detected enhancement up to 28-hours after FUS. Therefore, the finding that Vasculotide substantially reduces BBB permeability to intraperitoneal gadolinium, suggests that Vasculotide may have a potent effect on the restoration of vascular stability following FUS.

Additionally, it is important to note that gadolinium is relatively small (574 Da) compared to EB ( $70 \mathrm{kDa}$ in circulation), thus larger tracers may produce more rapid closure times and may not reflect the size of biological molecules (e.g. albumin 67-69 $\mathrm{kDa}$, globulins $>90 \mathrm{kDa}$ ) to which the BBB would be exposed [35]. Furthermore, the report by Sheikov et al. not only demonstrated that HRP and lanthanum chloride did not pass beyond the endothelial lining by 6-hours post FUS, but also that tight junctions were reformed after 6 hours [2]. This suggests that although the BBB may be permeable to gadolinium, the ability of large, potentially toxic molecules is rapidly diminished after FUS.

Previous studies have not detected overt edema, red blood cell extravasation or tissue injury after FUS $[4,5,30,32,35,43]$. Although Alonso et al. detected albumin in the brain parenchyma beginning 30 minutes post-FUS, this was phagocytized by glial cells and cleared from the brain as early as 1-hour and up to 24-hours post-FUS [44]. Similarly, the results presented here indicate extravasation fibrinogen, IgG and erythrocytes 24-hours post-FUS, particularly in the mice with prolonged BBB opening (Fig. 7). However, injection of EB at 24-hours post-FUS did not cross the BBB in either non-Tg or TgCRND8 mice (Fig. 3B), suggesting the aforementioned blood-borne molecules likely entered the parenchyma at an earlier time point. Previous work by Jordão et al. in TgCRND8 mice demonstrated elevated levels of endogenous antibodies (IgG, IgM) 4-days after FUS [11]. These endogenous antibodies co-localized with amyloid- $\beta$ plaques and could contribute to FUS-mediated plaque reduction $[11,14,43]$. Taken 
together, it is conceivable that 24-hours after FUS the BBB is impermeable to the entry of additional blood-borne molecules, while Vasculotide treatment reduced the initial entry and/or subsequent detection of blood-borne molecules.

The results presented here regarding the use of FUS to safely induce BBB permeability in TgCRND8 mice are consistent with previous studies by Jordão et al., and Burgess et al. $[11,39,43]$. Yet, we have demonstrated for the first time the applicability of Vasculotide in reducing BBB closure time after FUS. Our current study employed a chronic Vasculotide treatment, and it is possible that shorter treatment durations may be sufficient. Indeed, Vasculotide has been shown to reduce vascular permeability in peripheral organs using acute paradigms. As examples, treatment with Vasculotide 16- and 1-hour before, and 24 and 48-hours post cecal-ligation-andpuncture was sufficient to protect against vascular leak in sepsis [17]. Additionally, Vasculotide treatment 7-hours before a 70\% lethal dose of lipopolysaccharide administration protected against endotoxemic lung injury [22]. Vasculotide posttreatment has also demonstrated success against ionizing radiation damage [19] and influenza [21]. Future research using an acute Vasculotide treatment paradigm may also prove efficacious to restore BBB integrity.

Overall, our data demonstrates that Vasculotide is able to accelerate BBB restoration after FUS in the presence of amyloid pathology in the TgCRND8 mouse model of AD. These results indicate a potential role for Vasculotide as a treatment to promote BBB restoration in response to FUS-induced permeability. Furthermore, the impact of Vasculotide on the BBB could conceivably be extended to cases of stroke-as supported by a recent study from Venkat et al. in a preclinical model of stroke [18], neurodegenerative diseases and vascular disorders where the $\mathrm{BBB}$ is compromised.

\section{Limitations}

A limitation of our study is that we were unable to detect changes in the molecular components of the BBB in response amyloid pathology or Vasculotide. Using qRT-PCR we did not find significant differences in the transcript levels of ZO-1, occludin and claudin- 5 between TgCRND8 and non-Tg mice (Supp. Fig. 1). Furthermore, treatments with Vasculotide did not influence these BBB-related transcripts [45]. Additionally, we found that Tie1 and Tie2 transcript levels did not differ in TgCRND8 mice compared to non-Tg (Supp. Fig. 2A, B) [45]. The expression levels of Ang1, however, were higher in TgCRND8 compared to non-Tg mice (Supp. Fig. 2C)
[45]. With all being considered, we were unable to determine the mechanisms by which Vasculotide acts on the BBB to accelerate its restoration.

Future studies are required to characterize the effect of Vasculotide on the molecular and morphological vessel architecture, which was never done in the brain. At the level of cell junctions, in vitro and in mouse lung tissue, Vasculotide has been shown to increase VE-cadherin [22]. Vasculotide has also been shown to reduce TNF $\alpha$ in the brain [18], which is known to alter BBB permeability by upregulation of ICAM-1 and VCAM-1 [46]. Indeed, Vasculotide has also been demonstrated to reduce ICAM-1 and VCAM-1 levels [24,47]. Vasculotide has been shown to increase angiogenesis in wound healing assays [16]. It is known that TgCRND8 mice have increased arteriolar and capillary tortuosity and impaired vascular reactivity associated with cerebral amyloid angiopathy [7]. As such, it is plausible that Vasculotide treatment could alter the vessel architecture and plasticity which can contribute to the vascular response to FUS and modulation. Ultimately, there are still gaps in our understanding of how both FUS and Vasculotide modulate the brain vasculature. It is possible that Vasculotide initiates a cascade of events which ultimately result in the reduction of BBB permeability following FUS.

\section{Abbreviations}

FUS: focused ultrasound; BBB: blood-brain barrier; AD: Alzheimer's disease; CAA: cerebral amyloid angiopathy; VT: vasculotide; Ang-1: angiopoietin-1; PEG: polyethylene glycol; EB: Evans blue.

\section{Supplementary Material}

Supplementary methods and figures. http://www.medsci.org/v18p0482s1.pdf

\section{Acknowledgements}

We thank Drs. Paul Fraser, David Westaway, and Peter St George-Hyslop for their contributions in creating the TgCRND8 mice and providing breeding pairs used to establish our colony. The authors would like to thank Shawna Rideout and Alex Garces for their help with animal work. We acknowledge Melissa Theodore for breeding and genotyping of the mice used in this study. We thank Leya Aubert-Tandon for her assistance with Photoshop. The authors acknowledge Kairavi Shah for her expertise with the FUS procedures and Nathan McDannold for the MATLAB script use for enhancement analysis. Hang $\mathrm{Yu}$ Lin created the illustration for the graphical abstract. 


\section{Author Contributions}

ML performed experiments and analysis, prepared figures and wrote manuscript. $\mathrm{SH}$ performed histological analysis. KM assisted with experiments. MO performed ultrasound experiments and assisted with analysis. PV and DD provided Vasculotide. ML, DD, PV, KH and IA contributed experimental and analytical designs. All authors contributed to reviewing and editing the manuscript.

\section{Funding}

These experiments were supported by CIHR (FRN 137064, IA; FRN 119312, KH), Weston Brain Institute (IA), and NIH grant (R01-EB003268, KH), as well as the Canada Research Chair program (IA, KH, $\mathrm{MO})$.

\section{Competing Interests}

PVS \& DJD: are named inventors on grants and submitted patents related to Vasculotide. PVS also holds stock options within Vasomune. The other authors declare no competing financial interests.

\section{References}

1. Hynynen K, McDannold N, Vykhodtseva N, Jolesz FA. Noninvasive MR imaging-guided focal opening of the blood-brain barrier in rabbits. Radiology. 2001 Sep;220(3):640-6.

2. Sheikov N, McDannold N, Sharma S, Hynynen K. Effect of Focused Ultrasound Applied With an Ultrasound Contrast Agent on the Tight Junctional Integrity of the Brain Microvascular Endothelium. Ultrasound Med Biol. 2008 Jul;34(7):1093-104.

3. Deng J, Huang Q, Wang F, Liu Y, Wang Z, Wang Z, et al. The role of caveolin-1 in blood-brain barrier disruption induced by focused ultrasound combined with microbubbles. J Mol Neurosci. Humana Press Inc; 2012 Mar;46(3):677-87.

4. Choi JJ, Pernot M, Brown TR, Small SA, Konofagou EE. Spatio-temporal analysis of molecular delivery through the blood-brain barrier using focused ultrasound. Phys Med Biol. IOP Publishing: 2007 Sep 21:52(18):5509.

5. McDannold N, Arvanitis CD, Vykhodtseva N, Livingstone MS. Temporary Disruption of the Blood-Brain Barrier by Use of Ultrasound and Microbubbles: Safety and Efficacy Evaluation in Rhesus Macaques. Cancer Res. American Association for Cancer Research; 2012 Jul 15;72(14):3652-63.

6. McDannold N, Zhang Y. Ultrasound-mediated blood-brain barrier disruption for targeted drug delivery in the central nervous system. Proc SPIE. 2015;9467.

7. Dorr A, Sahota B, Chinta LV, Brown ME, Lai AY, Ma K, et al. Amyloid- $\beta$-dependent compromise of microvascular structure and function in a model of Alzheimer's disease. Brain. 2012 Oct;135(Pt 10):3039-50.

8. Zlokovic BV. Neurovascular pathways to neurodegeneration in Alzheimer's disease and other disorders. Nat Rev Neurosci. 2011 Dec;12(12):723-38.

9. Starr JM, Farrall AJ, Armitage P, McGurn B, Wardlaw J. Blood-brain barrier permeability in Alzheimer's disease: a case-control MRI study. Psychiatry Res. 2009 Mar 31;171(3):232-41.

10. Raymond SB, Treat LH, Dewey JD, McDannold NJ, Hynynen K, Bacskai BJ. Ultrasound Enhanced Delivery of Molecular Imaging and Therapeutic Agents in Alzheimer's Disease Mouse Models. Bush AI, editor. PLoS ONE. Public Library of Science; 2008 May 14;3(5):e2175

11. Jordão JF, Thévenot E, Markham-Coultes K, Scarcelli T, Weng Y-Q, Xhima K, et al. Amyloid- $\beta$ plaque reduction, endogenous antibody delivery and glial activation by brain-targeted, transcranial focused ultrasound. Exp Neurol. 2013 Oct;248:16-29.

12. Burgess A, Dubey S, Yeung S, Hough O, Eterman N, Aubert I, et al. Alzheimer disease in a mouse model: MR imaging-guided focused ultrasound targeted to the hippocampus opens the blood-brain barrier and improves pathologic abnormalities and behavior. Radiology. 2014 Dec;273(3):736-45.

13. Scarcelli T, Jordão JF, O'Reilly MA, Ellens N, Hynynen K, Aubert I. Stimulation of hippocampal neurogenesis by transcranial focused ultrasound and microbubbles in adult mice. Brain stimulation. NIH Public Access; 2014 Mar;7(2):304-7.

14. Leinenga G, Götz J. Scanning ultrasound removes amyloid- $\beta$ and restores memory in an Alzheimer's disease mouse model. Science Translational Medicine. American Association for the Advancement of Science; 2015 Mar $11 ; 7(278): 278 \mathrm{ra33}-3$.
15. Lipsman N, Meng Y, Bethune AJ, Huang Y, Lam B, Masellis M, et al. Blood-brain barrier opening in Alzheimer's disease using MR-guided focused ultrasound. Nat Commun. Nature Publishing Group; 2018 Jul 25;9(1):2336.

16. Van Slyke P, Alami J, Martin D, Kuliszewski M, Leong-Poi H, Sefton MV, et al. Acceleration of Diabetic Wound Healing by an Angiopoietin Peptide Mimetic. Tissue Eng Part A. 2009 Jun;15(6):1269-80.

17. Kümpers P, Gueler F, David S, Van Slyke P, Dumont DJ, Park J-K, et al. The synthetic Tie2 agonist peptide vasculotide protects against vascular leakage and reduces mortality in murine abdominal sepsis. Crit Care. 2011;15(5):R261.

18. Venkat P, Yan T, Chopp M, Zacharek A, Ning R, Van Slyke P, et al. Angiopoietin-1 Mimetic Peptide Promotes Neuroprotection after Stroke in Type 1 Diabetic Rats. Cell Transplant. 2018 Aug 20;:963689718791568.

19. Korpela E, Yohan D, Chin LC, Kim A, Huang X, Sade S, et al. Vasculotide, an Angiopoietin-1 mimetic, reduces acute skin ionizing radiation damage in a preclinical mouse model. BMC Cancer. BioMed Central Ltd; 2014;14(1):614.

20. Wu FTH, Lee CR, Bogdanovic E, Prodeus A, Gariépy J, Kerbel RS. Vasculotide reduces endothelial permeability and tumor cell extravasation in the absence of binding to or agonistic activation of Tie2. EMBO Mol Med. 2015 Apr 7.

21. Sugiyama MG, Armstrong SM, Wang C, Hwang D, Leong-Poi H, Advani A, et al. The Tie2-agonist Vasculotide rescues mice from influenza virus infection. Sci Rep. 2015;5:11030.

22. David S, Ghosh CC, Kümpers P, Shushakova N, Van Slyke P, Khankin EV, et al. Effects of a synthetic PEG-ylated Tie-2 agonist peptide on endotoxemic lung injury and mortality. Am J Physiol Lung Cell Mol Physiol. 2011 Jun;300(6):L851-62

23. Rübig E, Stypmann J, Van Slyke P, Dumont DJ, Spieker T, Buscher K, et al. The Synthetic Tie2 Agonist Peptide Vasculotide Protects Renal Vascular Barrier Function In Experimental Acute Kidney Injury. Sci Rep. 2016;6:22111.

24. Bourdeau A, Van Slyke P, Kim H, Cruz M, Smith T, Dumont DJ. Vasculotide, an Angiopoietin-1 mimetic, ameliorates several features of experimental atopic dermatitis-like disease. BMC Res Notes. BioMed Central; 2016;9(1):289.

25. Trieu M, van Meurs M, van Leeuwen ALI, Van Slyke P, Hoang V, Geeraedts LMG, et al. Vasculotide, an Angiopoietin-1 Mimetic, Restores Microcirculatory Perfusion and Microvascular Leakage and Decreases Fluid Resuscitation Requirements in Hemorrhagic Shock. Anesthesiology. 2018 Feb;128(2):361-74.

26. Gutbier B, Jiang X, Dietert K, Ehrler C, Lienau J, Van Slyke P, et al. Vasculotide reduces pulmonary hyperpermeability in experimental pneumococcal pneumonia. Crit Care. BioMed Central; 2017 Nov 13;21(1):274

27. Chishti MA, Yang DS, Janus C, Phinney AL, Horne P, Pearson J, et al. Early-onset Amyloid Deposition and Cognitive Deficits in Transgenic Mice Expressing a Double Mutant Form of Amyloid Precursor Protein 695. J Biol Chem. American Society for Biochemistry and Molecular Biology; 2001 Jun 15;276(24):21562-70.

28. Abrahao A, Meng $Y$, Llinas M, Huang $Y$, Hamani C, Mainprize T, et al. First-in-human trial of blood-brain barrier opening in amyotrophic lateral sclerosis using MR-guided focused ultrasound. Nat Commun. Nature Publishing Group; 2019 Sep 26;10(1):4373-9.

29. Meng $Y$, Abrahao A, Heyn CC, Bethune AJ, Huang $Y$, Pople $C B$, et al. Glymphatics Visualization after Focused Ultrasound-Induced Blood-Brain Barrier Opening in Humans. Annals of Neurology. John Wiley \& Sons, Ltd; 2019 Dec;86(6):975-80.

30. O'Reilly MA, Hough O, Hynynen K. Blood-Brain Barrier Closure Time After Controlled Ultrasound-Induced Opening Is Independent of Opening Volume. J Ultrasound Med. 2017 Jan 21.

31. O'Reilly MA, Hynynen K. A PVDF receiver for ultrasound monitoring of transcranial focused ultrasound therapy. IEEE Trans Biomed Eng. IEEE; 2010 Sep;57(9):2286-94.

32. O'Reilly MA, Hynynen K. Blood-brain barrier: real-time feedback-controlled focused ultrasound disruption by using an acoustic emissions-based controller. Radiology. 2012 Apr;263(1):96-106.

33. Choi JJ, Feshitan JA, Baseri B, Wang S, Tung Y-S, Borden MA, et al. Microbubble-size dependence of focused ultrasound-induced blood-brain barrier opening in mice in vivo. IEEE Trans Biomed Eng. 2010 Jan;57(1):145-54.

34. Jaffer H, Adjei IM, Labhasetwar V. Optical imaging to map blood-brain barrier leakage. Sci Rep. 2013;3:3117.

35. Samiotaki G, Konofagou EE. Dependence of the reversibility of focusedultrasound-induced blood-brain barrier opening on pressure and pulse length in vivo. IEEE Trans Ultrason Ferroelectr Freq Control. IEEE; 2013 Nov; 60(11):2257-65.

36. Marty B, Larrat B, Van Landeghem M, Robic C, Robert P, Port M, et al. Dynamic study of blood-brain barrier closure after its disruption using ultrasound: a quantitative analysis. J Cereb Blood Flow Metab. 2012 Oct:32(10):1948-58

37. Hynynen K, McDannold N, Sheikov NA, Jolesz FA, Vykhodtseva N. Local and reversible blood-brain barrier disruption by noninvasive focused ultrasound at frequencies suitable for trans-skull sonications. Neuroimage. 2005 Jan 1;24(1):12-20.

38. Hawkes CA, McLaurin J. Selective targeting of perivascular macrophages for clearance of $\beta$-amyloid in cerebral amyloid angiopathy. Proc Natl Acad Sci USA. National Acad Sciences; 2009 Jan 27;106(4):1261-6.

39. Burgess A, Nhan T, Moffatt C, Klibanov AL, Hynynen K. Analysis of focused ultrasound-induced blood-brain barrier permeability in a mouse model of Alzheimer's disease using two-photon microscopy. J Control Release. 2014 Oct 28;192:243-8. 
40. David S, Park J-K, Meurs MV, Zijlstra JG, Koenecke C, Schrimpf C, et al. Acute administration of recombinant Angiopoietin-1 ameliorates multiple-organ dysfunction syndrome and improves survival in murine sepsis. Cytokine. 2011 Aug;55(2):251-9.

41. Mei J, Cheng Y, Song Y, Yang Y, Wang F, Liu Y, et al. Experimental study on targeted methotrexate delivery to the rabbit brain via magnetic resonance imaging-guided focused ultrasound. J Ultrasound Med. 2009 Jul;28(7):871-80.

42. Konofagou EE. Optimization of the ultrasound-induced blood-brain barrier opening. Theranostics. 2012;2(12):1223-37.

43. Jordão JF, Ayala-Grosso CA, Markham K, Huang Y, Chopra R, McLaurin J, et al. Antibodies Targeted to the Brain with Image-Guided Focused Ultrasound Reduces Amyloid- $\beta$ Plaque Load in the TgCRND8 Mouse Model of Alzheimer's Disease. PLoS ONE. Public Library of Science; 2010 May 11;5(5):e10549.

44. Alonso A, Reinz E, Fatar M, Hennerici MG, Meairs S. Clearance of albumin following ultrasound-induced blood-brain barrier opening is mediated by glial but not neuronal cells. Brain Res. Elsevier B.V; 2011 Jul;1411:9-16.

45. Lynch M. Investigation of Vascular Function and its Modulation in a Mouse Model of Amyloidosis. [Doctoral dissertation, University of Toronto]. 2019: $1-143$.

46. Małkiewicz MA, Szarmach A, Sabisz A, Cubała WJ, Szurowska E, Winklewski PJ. Blood-brain barrier permeability and physical exercise. J Neuroinflammation. BioMed Central; 2019 Jan 24;16(1):15-6.

47. Thamm K, Njau F, Van Slyke P, Dumont DJ, Park J-K, Haller H, et al. Pharmacological Tie2 activation in kidney transplantation. World J Transplant. 2016 Sep 24;6(3):573-82. 